\title{
Effects of size and frequency dispersion in plasmonic cloaking
}

\author{
Andrea Alù ${ }^{1,2}$ and Nader Engheta ${ }^{1, *}$ \\ ${ }^{1}$ Department of Electrical and Systems Engineering, University of Pennsylvania, Philadelphia, Pennsylvania 19104, USA \\ ${ }^{2}$ Department of Electrical and Computer Engineering, University of Texas at Austin, Austin, Texas 78712-0240, USA
}

(Received 15 May 2008; published 27 October 2008)

\begin{abstract}
The plasmonic venue to realize invisibility and cloaking [A. Alù and N. Engheta, Phys. Rev. E 72, 016623 (2005)] is analyzed here in terms of its limitations and its frequency dispersion relative to the cloak size. Intrinsic limits due to causality and comparison with transformation-based cloaking techniques are discussed and analyzed. An interestingly simple low-dispersion cloak is also suggested for background materials with larger refractive index. These results may shed light on this scattering cancellation phenomenon, suggesting potential applications in scattering reduction and noninvasive probing.
\end{abstract}

DOI: 10.1103/PhysRevE.78.045602

Several suggestions for cloaking applications have been recently proposed in the technical literature, based on the exciting anomalous properties of complex media and/or properly engineered metamaterials [1-12]. At the moment arguably the most popular technique, transformation-based cloaking [1-4] has been recently realized experimentally in setups at microwave frequencies [2], achieving relevant scattering reduction by effectively shielding a given region of space with a properly designed metamaterial cloak that may bend the impinging electromagnetic wave around it at will. Although in principle this technique may work for any size of the cloaked region $[1,3]$, in reality some limitations may be forecasted for its practical realization, mainly due to the required electromagnetic response of the cloak materials, which should have specific inhomogeneity and anisotropy profiles both in their permittivity and permeability. In this sense, for their practical realization some approximations to the ideal theory are necessary and the cloak performances are consequently less than ideal, particularly for larger size cloaks. Moreover, such cloaking technique may be strongly dependent on frequency and on the field polarization.

As a different cloaking technique, earlier in 2005 we have introduced the idea of canceling the dominant portion of scattering from a given object by employing plasmonic materials with low positive permittivity. In this sense, readily available or easily realizable plasmonic materials may be utilized, with no specific or particular profiles of inhomogeneity or anisotropy, and cloaking may be achieved in three dimensions (3D), and for all polarizations and angles of incidence [6]. The inherently nonresonant features of this cloaking technique make it relatively more robust to frequency, geometry, and design variations [7]. Relatively simple realizations with metamaterial technology have been envisioned at microwave frequencies in [8]. Moreover, arbitrary collections of dielectric and/or conducting objects may also be simultaneously cloaked [9] and the use of multiple layers may further provide multifrequency operation and larger scattering reduction [10]. Despite its advantages, this cloaking technique has often been assumed to be limited to small-sized objects, much smaller than the wavelength of operation, and to not represent an actual "cloaking" mechanism, due to the

\footnotetext{
*Corresponding author; engheta@ee.upenn.edu
}

PACS number(s): 42.70.-a, 42.79.-e, 42.50.Gy, 33.20.Fb

general dependence of the cloak design on the properties of the object to be cloaked. In the following, we aim to address these assumptions and issues and to provide some insights into this plasmonic cloaking mechanism, also with comparison to transformation-based cloaks. Moreover, our findings in this paper inspire the realization of a low-dispersion, relatively broadband cloaking mechanism, which may be realizable when the background material has an index of refraction relatively large.

Consider the geometry in the inset of Fig. 1, i.e., a perfectly conducting spherical object with diameter $2 a=1.5 \lambda_{0}$, with $\lambda_{0}$ being the wavelength of free space at the (arbitrary) design frequency $f_{0}=1 \mathrm{GHz}$. The size of this object is not electrically small, and in fact multiple Mie scattering orders contribute to the "visibility" of the object when illuminated by an impinging plane wave. However, following [6-10], the presence of a suitably designed homogeneous layer with low permittivity supports polarization currents that may, due to its negative polarizability, cancel a major portion of the scattering radiation from the object, making the system less visible to its surrounding.

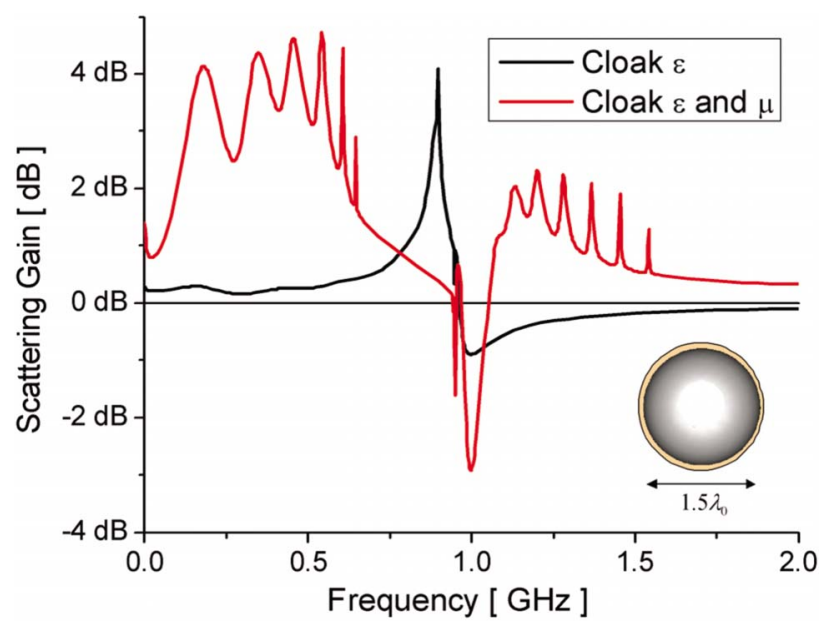

FIG. 1. (Color online) Scattering "gain" from a conducting particle with diameter $2 a=1.5 \lambda_{0}$ using two different cloaking designs. The scattering gain is obtained as the ratio of the total scattering cross section of the overall object with its cloak, divided by the total scattering cross section of the sphere alone. 
It is noted that, even if in [6-10] we were mainly concerned with canceling the dipolar contribution to the scattering, which is dominant for relatively small objects, in principle this technique may be applied to any dominant scattering order (i.e., multipole) by properly selecting and designing the cover geometry. It is found that in order to minimize the contribution of $T M$ spherical harmonics (those associated with electrical multipoles) to the scattered field from the conducting sphere a low permittivity cover is required, consistent with our previous findings. In order to additionally suppress the residual TE (magnetic) multipoles, permeability greater than unity is sufficient, since conducting objects are usually characterized by a negative magnetic polarizability.

Figure 1 reports the scattering "gain," defined as the ratio (in $\mathrm{dB}$ ) between the total scattering cross section of the cloaked object divided by that of the bare sphere. Negative values in $\mathrm{dB}$ clearly correspond to scattering reduction. The figure refers to two designs for the cloak: One (black darker line) constitutes a simple Drude material with permittivity,

$$
\varepsilon_{c}(f)=\varepsilon_{0}\left\{1-f_{p}^{2} /[f(f+i \gamma)]\right\},
$$

where $f_{p}=0.95 f_{0}$ and $\gamma=10^{-3} f_{0}$ taking into account losses and frequency dispersion of the plasmonic material and designed to have $\varepsilon_{c}\left(f_{0}\right)=0.1 \varepsilon_{0}$, with an optimized radius $a_{c}$ $=1.01 a$; the second (red lighter line) assumes the same permittivity dispersion, but with an additional optimized permeability $\mu_{c}=5.96 \mu_{0}$ and optimized radius $a_{c}=1.058 a$. It is evident that at the design frequency both cloaks may provide a minimized scattering cross section, with reduction of visibility as compared with the uncloaked case. This is obtained with a simple homogeneous and isotropic thin layer covering the conducting object, despite the large number of contributing multipole harmonics [in the bare geometry, the electric dipole contribution is responsible for only $12 \%$ of the total scattering and the total number of non-negligible scattering orders (i.e., multipoles) is over 30]. Figure 1 indeed shows that this cloaking technique may be able to suppress simultaneously contributions for several scattering multipoles achieving a 50\% scattering reduction, even for this relatively large geometry. It is interesting to notice that this optimized cloak does not completely cancel any of the relevant scattering multipoles, but instead minimizes the total scattering cross section from the object in all directions by reducing the magnitudes of several of these multipoles. In this sense, its behavior is quite different from the cloaks presented in [6-10] for relatively smaller objects, for which the scattering was dominated by one or two scattering orders, on which the cloaking technique was focused.

As discussed in recent works [11,12], causality implies that even an "ideal" cloak may be able to substantially suppress the scattering only over a very limited bandwidth, which drastically decreases when the size of the cloak is increased. This bandwidth narrowing is also associated with a necessary stronger sensitivity to the design parameters when the size of the cloaked region is increased. This is consistent with Fig. 1, where a scattering reduction of about $1 \mathrm{~dB}$ may be achieved with a cloak with only low positive permittivity (and $\mu_{c}=\mu_{o}$ ) over a relatively broad bandwidth,

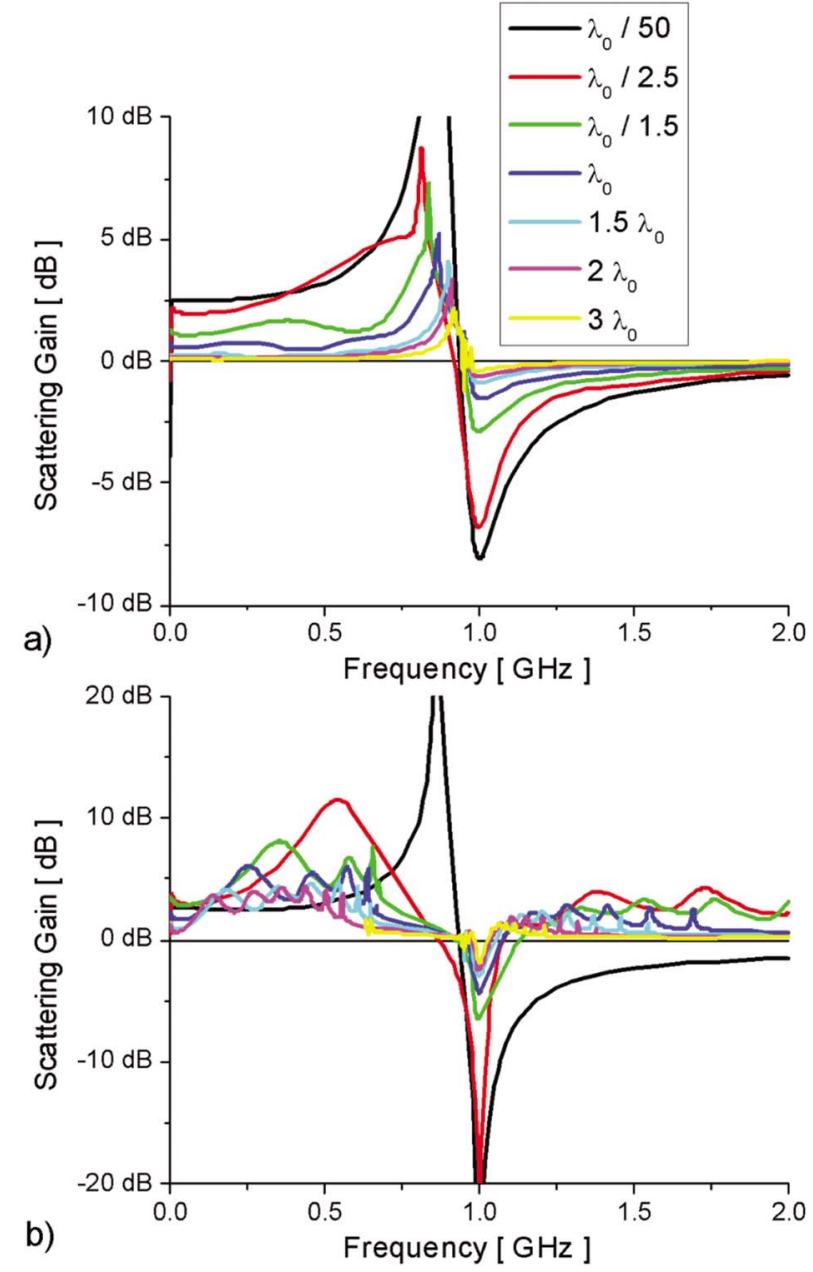

FIG. 2. (Color online) Scattering gain from a conducting particle, varying its diameter as indicated in the legend, for a cloak with permittivity given in Eq. (1) and (a) free-space permeability, (b) optimized positive permeability in order to cancel the residual scattering. For each curve the cloak outer radius has also been optimized for achieving minimum scattering at frequency $f_{0}$.

whereas a larger scattering reduction may be obtained with $8 \%$ fractional bandwidth for the case of the cloak with both optimized $\varepsilon_{c}$ and $\mu_{c}$.

Ideally, a transformation-based cloak [1] may achieve a larger reduction in scattering at the design frequency. However, the sensitivity to stringent inhomogeneity and anisotropy profiles of the cloak makes these ideal results challenging to achieve in practice. In fact, the amount of scattering reduction achieved with the simple isotropic and homogeneous layer of Fig. 2 is comparable with the scattering reduction obtained in the simulations for the cylindrical setup presented in [2], for roughly the same electrical size of the object, which we have resimulated with finite element software [13] using the suggested parameters in order to extract the scattering width reduction (its value was not indicated in the original paper [2]). However, the bandwidth of operation, when considering a Lorentzian dispersion for the radial component of the permeability tensor in the scenario of [2] is substantially smaller than in this case, confirming the results of [12] that show how a relatively larger cloaking bandwidth 
is achievable by slightly increasing the level of minimum scattering.

In a fair comparison between the results in Fig. 1 and the transformation-based cloaking technique, several points should be underlined: (a) The method discussed here requires an arguably much simpler cloak design, constituted of a simple homogeneous and isotropic plasmonic material, in some case (but not always) with some magnetic properties; (b) the homogeneity and isotropy of the cloak has been chosen here for the sake of simplicity and it is not a constraint, as shown in the metamaterial design presented in [8] using this cloaking technique and using an anisotropic metamaterial for convenience; (c) here the cloak is much thinner, occupying an overall smaller region of space and therefore the causality constraints over the limitations in bandwidth may lead to a less narrow band; (d) the geometry analyzed here is $3 \mathrm{D}$, and therefore the scattering reduction happens on both planes of polarization, all over the incident angles. In the simulations and experiments in [1-5], the cloaked objects were cylindrical and the reduction of cross section was therefore limited to one single plane and field polarization; (e) the results in Fig. 1 are relatively robust to variations in the design parameters, being based on scattering cancellation [7]. In other words, the design parameters presented here do not have to be chosen exactly and thus deviations from these ideal spherical shapes or from the optimized electromagnetic parameters would not sensibly affect the cloaking performance [7]; (f) the use of multilayered cloaks may further increase the performance of this technique, as suggested in [10]; (g) this technique may also be applied to dielectric objects, for which the impinging electromagnetic wave is allowed to penetrate the cloaked region, relaxing the stringent "rerouting" constraints and therefore further improving bandwidth of operation, robustness to size increase, and overall scattering reduction. Regarding this last point, it should indeed be emphasized that here we are reporting the most challenging situation for this cloaking technique, i.e., the case in which the object to be cloaked is impenetrable. When dielectric objects are considered, or at infrared and optical frequencies where metals are plasmonic in nature, the wave may partly or totally penetrate the object, allowing a substantial improvement over the present results.

Figure 2 shows the variation of the scattering "gain" as a function of the particle size, for the two types of cloak analyzed in Fig. 1. For small particles, for which a dominant electric and magnetic dipolar contribution is expected, the nonmagnetic cloak [Fig. 2(a)] is already capable of suppressing most part of the scattering from the particle, and the addition of suitably designed magnetic properties suppresses further the residual visibility. When the diameter of the conducting sphere is increased the relative scattering cancellation is reduced, but still relevant scattering reduction may be achieved, larger when a magnetic cloak is considered. Consistently with the previous discussion, the corresponding bandwidth is also reduced for larger objects.

Table I reports the optimized parameters utilized in evaluating the different curves of Fig. 2, confirming the fact that, despite the electrical size of the object to be cloaked, the optimized layer is only a few percents of the thickness of the object and in the magnetic case the layer has a permeability
TABLE I. Optimized parameters that minimize the total scattering cross section in the different geometries of Fig. 2. The column $a_{c} /\left.a\right|_{\mu_{c}=\mu_{0}}$ refers to the optimized radius for the only-permittivity cloak, whereas the next two columns contain the optimized permeability and radius for the cloak with also magnetic properties. In all of the examples $\varepsilon_{c}$ follows Eq. (1).

\begin{tabular}{lccc}
\hline \hline & $a_{c} /\left.a\right|_{\mu_{c}=\mu_{0}}$ & $\mu_{c}$ & $a_{c} /\left.a\right|_{\mu_{c}>\mu_{0}}$ \\
\hline$\lambda_{0} / 50$ & 1.1 & 5 & 1.1 \\
$\lambda_{0} / 2.5$ & 1.088 & 5.07 & 1.14 \\
$\lambda_{0} / 1.5$ & 1.049 & 3.71 & 1.145 \\
$\lambda_{0}$ & 1.025 & 5.02 & 1.093 \\
$1.5 \lambda_{0}$ & 1.01 & 5.96 & 1.058 \\
$2 \lambda_{0}$ & 1.006 & 6.64 & 1.049 \\
$3 \lambda_{0}$ & 1.0026 & 7.33 & 1.035 \\
\hline \hline
\end{tabular}

$\mu_{c}>\mu_{0}$. We reiterate that reasonable deviations from these optimized values would not sensibly affect the performance of the cloak [7].

It is interesting to note that the designs in Figs. 2 and 3 may be fully considered "cloaks" in the same manner and meaning as those in different techniques [1-5] for metamaterial cloaking: The inner conducting object in the previous simulations may in fact represent a thin conducting shield,
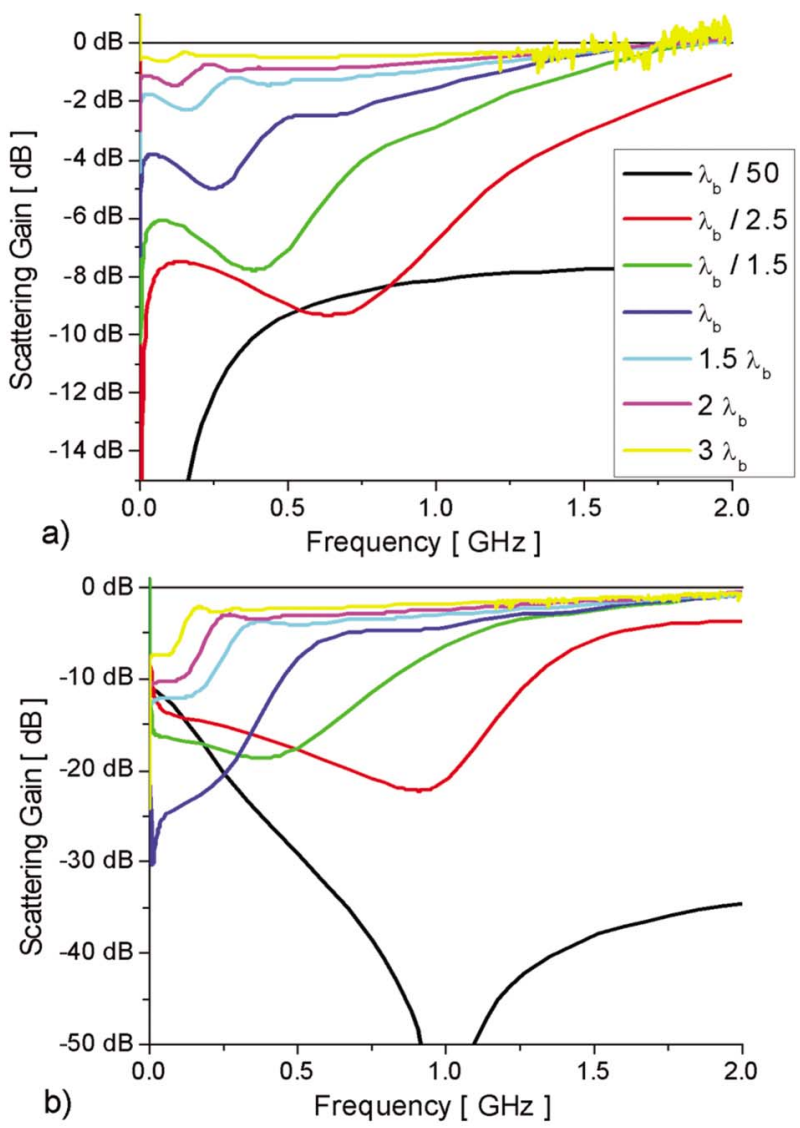

FIG. 3. (Color online) Similar to Fig. 2, but for a background material with permittivity $\varepsilon_{b}=10 \varepsilon_{0}$. In this case, the cloak permittivity is simply the same as free-space $\varepsilon_{0}$ with no material dispersion. Note the large bandwidth of scattering reduction. 
inside which the electromagnetic fields do not penetrate, therefore creating an isolated (3D) region of space where any object of smaller size may be inserted to be cloaked. This would be no different from the setups proposed in the transformation-based cloaking, where the inner layer of the anisotropic and inhomogeneous cloak is always required to have an effective permittivity or permeability being identically zero or infinite. In terms of materials, such inner layers are exactly equivalent to magnetic or electric conductors, which once again have the properties to completely shield the inner region from the impinging electromagnetic wave. The present setup, however, does not require any forms of anisotropy or inhomogeneity (although it can be anisotropic and/or inhomogeneous, if needed) and achieves analogous results in an arguably simpler way and in 3D.

Related to this analogy, as mentioned above, the technique presented here does not require the presence of a conducting shield. In fact, in several of our designs in [6] we have proposed analogous cloaks designed for dielectric (nonconducting objects). In other words, if the object to be cloaked is known, it is not necessary to first shield it with a conducting material, which would generate additional scattering, and then design a suitable cover for the conducting shield that effectively is what the transformation-based cloaks do. A more relevant design here may be obtained by directly designing the cover to "hide" the dielectric object of interest. It is noticed that in this scenario the electromagnetic wave may indeed penetrate the object, but this causes no harm as long as the overall system does not produce any noticeable scattering in the outside region. In fact, wave penetration inside the dielectric object in the plasmonic cloaking has several positive effects: Since part of the wave energy can penetrate though the dielectric object, the requirement for total rerouting of the energy around the object is somewhat relaxed and the bandwidth of operation is consequently enlarged. Also, the residual scattering from the system is correspondingly decreased and often simpler nonmagnetic cloaks may be sufficient to drastically suppress the overall scattering. Moreover, such wave penetration may also be exploited for applications in noninvasive measurements and probing, since the field in the cloak may be accessible, while still the scattering from the overall system is essentially eliminated. We are currently working on these scenarios. Our simulations for the case of a dielectric sphere (not shown here) of the same size as the conducting sphere in Fig. 2 indeed show noticeably increased bandwidth of operation and further scattering reduction with respect to the conducting case.

Returning to the issue of bandwidth, it is interesting to point out that this cloaking technique is based on an integra- tive effect of scattering cancellation, and therefore it works in a quasistatic local scenario. This implies that the main limitations imposed by causality on the bandwidth are mainly associated with the required permittivity dispersion of plasmonic materials. This explains, for instance, the large scattering peaks in Fig. 2, which are associated with the plasmonic resonances of the cover at frequencies for which the Drude model (1) provides $\varepsilon_{c}<0$.

However, we may envision a scenario for which the cloaking material is not required to be dispersive, that is when the background has permittivity $\varepsilon_{b}>\varepsilon_{0}$. Consider for instance a background material with relative index of refraction around 3, which may represent, e.g., some liquid. In this case, in order to cloak analogous conducting particles as those considered in Fig. 2, it is not anymore necessary to employ a plasmonic material with low positive permittivity, since even a free-space cloak (e.g., an "air cloak") with permittivity $\varepsilon_{0}$ would be able to provide a local negative polarizability. In this case, the associated material dispersion is evidently totally avoided. As an example, Fig. 3 reports the dispersion of the scattering gain for similar particles as in Fig. 2 immerged in a background with $\varepsilon_{b}=10 \varepsilon_{0}$, and with a simple free-space "buffer" around them, envisioning the simplest imaginable cloak. (Notice that here their relative size is scaled down by about 3 times, since $\lambda_{0}$ is substituted by $\lambda_{b}$ $=\lambda_{0} / \sqrt{\varepsilon_{b} / \varepsilon_{0}}$, i.e., the background wavelength.) A proper optimization of the "cloak" radius (with the same values as reported in Fig. 1, since the problem is analogous at the design frequency $f_{0}$ ) shows that it is indeed possible to achieve a large scattering minimization with very weak frequency dispersion, valid also for relatively large particles. Allowing some magnetic properties for this dispersionless cloak may further reduce the visibility of the particles, still keeping the cloaking bandwidth extremely large. Even though it is understood that a high-index background may not be feasible for some applications of interest, this cloak may have interesting applications in marine or biological scenarios and in acoustic cloaking.

To conclude, we have discussed and analyzed here the effects of the object size on the dispersion and scattering reduction of the plasmonic cloaking technique [6-10], also drawing some comparisons with the current transformationbased cloaks. The results may have applications in camouflaging, scattering reduction, biological and medical measurements. We have also envisioned the possibility of a lowdispersion cloak when the refractive index of the background is larger than free space, which suggests that frequency dispersion of this cloaking technique is mainly due to the cloak's material properties.
[1] J. B. Pendry et al., Science 312, 1780 (2006).

[2] D. Schurig et al., Science 314, 977 (2006).

[3] U. Leonhardt, Science 312, 1777 (2006).

[4] W. Cai et al., Nat. Photonics 1, 224 (2007).

[5] G. W. Milton and N. A. P. Nicorovici, Proc. R. Soc. London, Ser. A 462, 3027 (2006).

[6] A. Alù and N. Engheta, Phys. Rev. E 72, 016623 (2005).
[7] A. Alù and N. Engheta, Opt. Express 15, 3318 (2007).

[8] M. G. Silveirinha et al., Phys. Rev. E 75, 036603 (2007).

[9] A. Alù and N. Engheta, Opt. Express 15, 7578 (2007).

[10] A. Alù and N. Engheta, Phys. Rev. Lett. 100, 113901 (2008).

[11] D. A. B. Miller, Opt. Express 14, 12457 (2006).

[12] H. Chen et al., Phys. Rev. B 76, 241104(R) (2007).

[13] COMSOL MultiPhysics 3.4, http://www.comsol.com/ 